\title{
Self-Assembly of Colloidosome Shells on Drug-Containing Hydrogels
}

\author{
Rachel T Rosenberg, Nily Dan
}

Department of Chemical and Biological Engineering, Drexel University, Philadelphia, Pennsylvania 19104 Email: dan@coe.drexel.edu

Received August 30 ${ }^{\text {th }}$, 2010; revised September 22 ${ }^{\text {nd }}$, 2010; accepted September $28^{\text {th }}, 2010$.

\begin{abstract}
Colloidosomes are composed of an aqueous or hydrogel corethat is coated by a semi-permeable colloidal shell. The properties of the shell can be varied to control the rate of release of encapsulated components such as drugs. Specifically, the pores formed between the colloidal particles suppress transport of large components, while allowing diffusion of smaller ones. Self-assembly of colloidal particles on hydrogel films is a convenient method forcolloidosome synthesis, but to date little is known regarding the effect (if any) of the encapsulated drug on the shell packing density. In this paper we examined self-assembly of colloidal shells on alginate films containing four model drugs: aspirin, caffeine, theophylline and theobromine. We find that the packing density in the colloidal shells is low for all drugs, and ranges between 0.16 and 0.3. There is no clear correlation between drug properties (in particular, water solubility) and the packing density of the self-assembled colloidal shell.
\end{abstract}

Keywords: Drug delivery, Diffusion, Colloids, Colloidosomes

\section{Introduction}

Selective control of drug transport is of interest for pharmaceutical, cosmetic, and food applications [1-6]. Colloidosomes [7] are aqueous microcapsule volumes coated by a shell of colloidal particles (see Figure 1) [7-16]: The packed colloidal particles form a barrier for transport, whose pore size may be controlled through choice of the colloidal particle size and the packing density in the shell [7-16]. As a result, colloidosomes allow size exclusion, namely, inhibition of transport of components whose size is larger than a critical value, while allowing transport of smaller molecules.

Several methodologies have been developed to form the colloidal shells of colloidosomes. Typically, they utilize the tendency of the particles to assemble at the interface between hydrophilic and hydrophobic fluids $[7,13,14,15]$. Although effective, the conditions involved are often not compatible with those needed for biological agents. As a result, they cannot be used for drug encapsulation.

Kim, et al [11] utilized a different method for colloidosome formation: The colloidal particles in the shell are driven to self-assemble on the surface of a hydrogel core by electrostatic interactions. The assembly is conducted in one step, and under benign environmental condition (i.e. aqueous solutions- including, potentially, serum, at room or body temperature).

We recently studied the release of model drugs from hydrogels coated by self-assembled colloidal monolayers $[17,18]$. Quite surprisingly, we found that the release profiles for each drug were insensitive to the size of the particles in the shell, for p[articles ranging from $20 \mathrm{~nm}$ to $3.3 \mu \mathrm{m}$ [17]. To understand these results, we adapted a two-film diffusion model[18], where the hydrogel is taken to be one film, and the shell coating the second film. The shell is modeled as a composite of an impenetrable phase (the colloidal particles) and a penetrable one that is similar in properties to the aqueous or hydrogel core (see Figure 1). The model finds that in the case of a loosely packed monolayer such as that of the self assembled shell, the release profile is indeed independent of the colloidal particle size, a function of three parameters only: the diffusion coefficient of the drug in the hydrogel core, the dimension (thickness) of the hydrogel core, and the packing density of particles in the shell. Thus, controlling the release rate of a drug from a colloidosome may be achieved through control of the shell packing density (since the diffusion coefficient is set by the hydrogel properties, and core size is typically set by the specific system needs). 


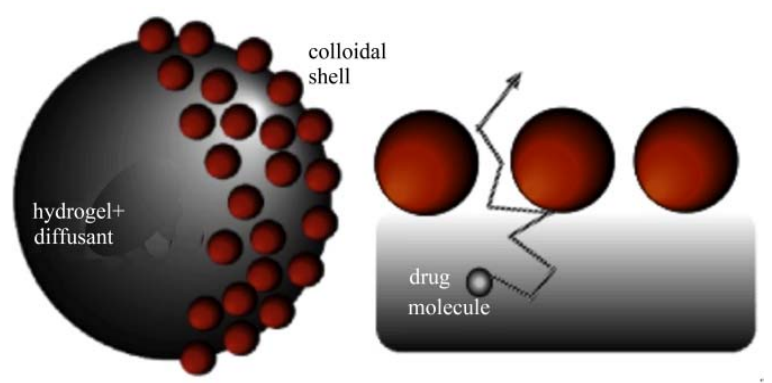

Figure 1. A schematic of the system examined. Left: A hydrogel containing a diffusant, or drug, is coated by a self-assembled colloidal monolayer, formed through adsorption of colloidal particles onto the oppositely charged hydrogel. Due to the self-assembly process, the shell is loosely packed, typically with a colloidal volume fraction of order $1 / 4$. The colloidal shell may be taken to be a composite of an impenetrable phase (the particles) and a penetrable one (hydrogel or aqueous solution). Right: The two-film diffusion model used takes the hydrogel to be one film, and the colloidal shell the other. The drug diffuses through the hydrogel until reaching the interface with the colloidal shell. It cannot penetrate through the particles, and must go through the pores in between the colloids, so that the rate of release is reduced when compared to the uncoated hydrogel.

Fitting our experimental results to the theoretical model [18] yielded that the packing density in the self-assembled colloidal shells is independent of the colloidal particle size. Moreover, it was similar for caffeine and aspirin, despite the differences in their chemical structure. This observation is somewhat surprising, since it has been previously found that the properties of a drug can significantly affect the properties of the encapsulating hydrogel, and thus the release profile: For example, studies of drug release from biodegradable polymeric films have clearly demonstrated that the drug properties can significantly affect the degradation rate, and thus release profile $[19,20]$. It may be expected that the presence of a drug in the hydrogel would affect the adsorption, and thus packing, of the colloidal particles.

To determine whether drug properties affect the packing density of a colloidal shell, and thus the release profile of the drug, we compared systems containing four model drugs: caffeine, theophylline, theobromine and aspirin. The first three are similar in structure, and even cause similar physiological effects (stimulation of the central nervous system and gastric acid secretion) [21]. Yet, they differ significantly in their solubility in water. The solubility of caffeine is $21.7 \mathrm{mg} / \mathrm{mL}$ [22], theophylline is $8 \mathrm{mg} / \mathrm{mL}$ [22], and theobromine is $0.5 \mathrm{mg} / \mathrm{mL}$ [23]. All drugs, including aspirin, have similar MWs (180 for aspirin, theophylline and theobromine, and 194 for caffeine), although aspirin differs from the three drugs in its chemical structure. The solubility of aspirin in water has an intermediate value similar to that of theophylline, of order $3-10 \mathrm{mg} / \mathrm{ml}$ [22].

It is somewhat difficult to predict, a-priori, what effect would drug properties have on the self-assembled colloidal shell. Self-assembly in these systems is driven by electrostatic interactions between charged groups on the hydrogel surface, and oppositely charged ones on the colloidal particles. It is possible that a hydrophobic drug (as determined by low solubility in water) may cause the hydrogel to collapse, thereby reducing the availability of the hydrogel surface groups to bonding with the colloidal particles. On the other hand, highly hydrophilic drugs may interact with the charged surface groups, thereby reducing particle adsorption. Regardless of the mechanism, it is expected that if the encapsulated drug has an effect on shell formation (and thus on the rate of drug release), the packing density of the colloidal particles in the shell would scale in some manner with drug solubility in aqueous solutions.

We find that the two-film model fit the release rate of all drugs well, for both uncoated and colloidal-shell coated hydrogels. However, the effect of the encapsulated drugs on the packing density of the shells is found to be weak: In all systems, the packing density of the colloids in the shell (namely, the volume fraction of particles) was calculated to be in a narrow range of 0.16-0.3. The packing density did not vary systematically with the drug properties, and in particular did correlate with the drug's water solubility. Indeed, the packing density for theobromine, which had the lowest solubility of all the drugs tested, was the lowest ( 0.16). However, the highest packing density was found for theophylline $(\sim 0.3)$, which has intermediate water solubility.

\section{Materials and Methods}

\subsection{Materials}

Sodium alginate, calcium chloride, aspirin, caffeine, theobromine and theophylline were purchased from Sigma-Aldrich (St. Louis, MO and Milwaukee, WI, USA). $3.3 \mu \mathrm{m}$ amidine $(\mathrm{C}(\mathrm{NH} 2)=\mathrm{NH}+)$ functionalized polystyrene particles were purchased from Invitrogen Molecular Probes. All reagents were used as purchased with no further purification.

\subsection{Synthesis of Hydrogel Films}

To allow consistent measurements of transport through a colloidal shell, the system consisted of an alginate hydrogel film (rather than spherical drops) impregnated with a given concentration of the chosen drug. The film was then coated by a monolayer of colloidal particles, and transport determined by measurement of the drug concentration in the medium surrounding the film 
$[17,18]$. Unlike transport from microgel colloidosomes, this geometry allows exact control over the system parameters (e.g. drug content in the hydrogel). The surrounding medium was kept, for all four drugs, at a concentration that is well below the relevant solubility limit even at $100 \%$ release.

A $2 \%$ (weight/volume) sodium alginate in water solution was shaken and sonicated to remove any excess air. $1 \mathrm{mg}$ of a model molecule was dissolved in $400 \mu \mathrm{L}$ of the sodium alginate solution and placed in a $30 \mathrm{~mL}(25 \mathrm{~mm}$ x $95 \mathrm{~mm}$ ) glass vial. The vials were placed in a convection oven at $40^{\circ} \mathrm{C}$ overnight to evaporate the excess solvent. $500 \mu \mathrm{L}$ of a $10 \%$ wt. solution of calcium chloride in water was added to the alginate and allowed to crosslink for 5 minutes. Excess calcium chloride was removed and polystyrene particles were added to the hydrogel film and incubated overnight. The films were subsequently washed with deionized water.

\subsection{Ultraviolet Spectroscopy}

Concentrations of molecules released were calculated using a linear calibration curve. 8 samples were made with pure water as the lowest concentration. The absorption spectra showed peaks for the following drugs: caffeine at $273 \mathrm{~nm}$, aspirin at $275 \mathrm{~nm}$, theophylline at $271 \mathrm{~nm}$ and theobromine at $272 \mathrm{~nm}$. All further measurements were taken at respective peak wavelengths. Concentrations were in the linear regime to minimize error.

\subsection{Diffusion Model}

The hydrogel-colloidal shell system is modeled as a two-film system [18], where the hydrogel is one film and the colloidal shell the other (see Figure 1). The transport of drug in such two-film systems is defined by three parameters: The hydrogel film thickness, a, the diffusion coefficient of the drug in the hydrogel, $\mathrm{D}$, and a dimensionless parameter $\mathrm{L}$ that accounts for the shell permeability: L is infinity when there is no shell (or when the shell does not offer resistance to transport), and zero when the shell is completely impenetrable to the drug [18]. The fraction of drug released at time $t$ is given in this approach by

$$
f(t)=1-\sum_{n=1}^{\infty} \frac{e^{-D \beta_{n}^{2} t / a^{2}}}{\beta_{n}^{2}\left(\beta_{n}^{2}+L^{2}-L\right)} / \sum_{n=1}^{\infty} \frac{1}{\beta_{n}^{2}\left(\beta_{n}^{2}+L^{2}-L\right)}
$$

where $\beta_{\mathrm{n}}$ are the roots of the expression [18]

$$
\beta_{n} \cot \beta_{n}+L-1=0
$$

Modeling the colloidal shell as a composite of an impenetrable phase (the colloidal particles) and a penetrable one yielded, in the case of a colloidal monolayer, the following relationship for L [18]:

$$
L \approx \pi(1-\varphi) / 2 \varphi\left(1+\pi \varphi^{2 / 3} / 6^{1 / 3}\right)
$$

where $\varphi$ is the volume fraction of colloids in the shell. Equation (3) is appropriate only for a loosely packed colloidal shell (where the packing density of the particles in the shell is less than $\sim 0.5$ ) and where the hydrogel core thickness $a$ is much larger than the colloidal particle shell thickness. The thickness of the monolayer colloidal shell is equal to the diameter of the colloidal particles which in this case, is much smaller than the thickness of the hydrogel film.

From (1-3) we see that the transport of a drug through a colloidal shell coating a hydrogel is expected to depend only on the hydrogel core thickness a, the diffusion coefficient through the hydrogel $\mathrm{D}$, and the colloidal packing density (volume fraction) in the shell $\varphi$. It should not vary with the particle diameter, as indeed verified by our previous experiments [17,18].

\section{Results and Discussion}

Our earlier work $[17,18]$ suggests that the release profile of drugs from hydrogels coated by a (loosely packed) self-assembled colloidal shell depends only on three parameters: The hydrogel film thickness, the diffusion coefficient of the drug in the hydrogel, and the packing density of the particles in the shell. Therefore, to determine the diffusion coefficient of the drugs in our alginate gels, we measured the release of the four drugs from uncoated hydrogels, as shown in Figure 2.

Literature values were published for the diffusion coefficient of some of the drugs in water [25,26]. However, those values cannot be directly applied to transport of these drugs through other media - such as our hydrogel. Moreover, although it might have been assumed that the relative ratios of the diffusion coefficients would be independent of the type of media (namely, if diffusion coefficient of one drug in water is higher than that of a second one, the same could be said of their rates in other aqueous-based media), studies show that this is not the case: For example, in water, caffeine and theophylline have similar diffusion coefficients [25,26]. Additionally, their release rate from alginate gels [24] and through Buccal tissue [27] has been found to be similar as well. Yet, in other media- transdermal tissue [27] and pig ear skin [28]- the transport rate of caffeine was found to be two orders of magnitude faster than that of theophylline.

Besides the medium, the specific formulation also affects the absolute and relative rates of the transport of different drugs [27,28].

In our case, the formulation of the hydrogel used for 


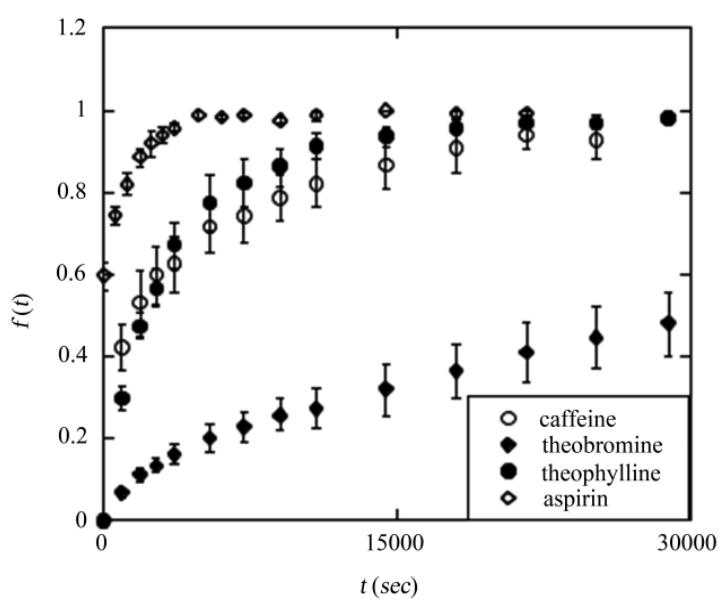

(a)

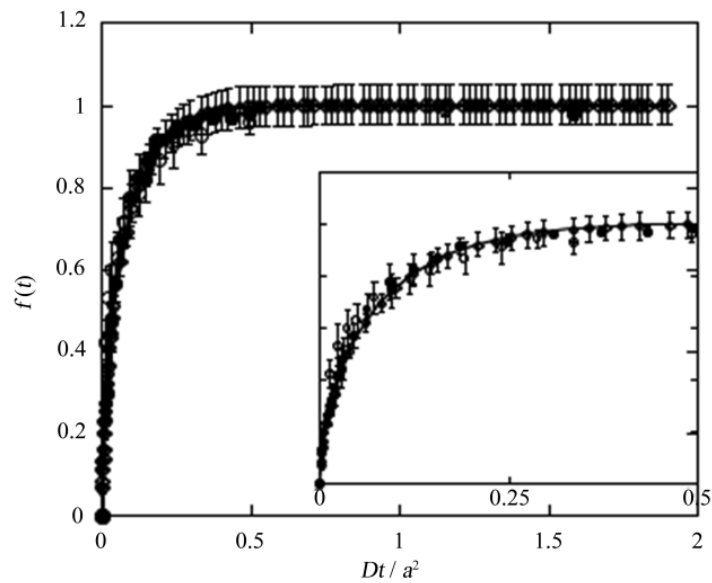

(b)

Figure 2: The release profile of the drugs from uncoated alginate hydrogels. $f$ defines the fraction of the initially encapsulated drug, released by time $t$. (A) The fractional release of drug as a function of time. The rate of aspirin release is fastest. Caffeine and theophylline have similar rates, and theobromine is much slower;(B) Fitting the release data to the diffusion model (Equations 1-2). Time is normalized by a factor of $a^{2} / D$, where $a$ is the thickness of the alginate film and $D$ the diffusion coefficient of the drug. The solid line describes the model prediction for uncoated films. The values of the normalization factor used in the fit are listed in Table 1.

Table 1. Drug and Model data.

\begin{tabular}{lcccccc}
\hline Drug & MW & $\begin{array}{c}\text { Solubility } \\
(\mathrm{mg} / \mathrm{ml})\end{array}$ & $a^{2} / D^{*}$ & $\begin{array}{c}D^{* *} \\
\left(\mathrm{~cm}^{2} / \mathrm{s}\right)\end{array}$ & $L_{*}^{* *}$ & $\phi^{*}$ \\
\hline Aspirin & 180 & $3-10[22]$ & 12,000 & $2.5 * 10^{-5}$ & 3.5 & 0.2 \\
Caffeine & 194 & $21.7[22]$ & 75,000 & $3.3 * 10^{-6}$ & 3.5 & 0.2 \\
$\begin{array}{c}\text { Theophyl- } \\
\text { line }\end{array}$ & 180 & $8[22]$ & 83,000 & $3 * 10^{-6}$ & 2 & 0.3 \\
$\begin{array}{l}\text { Theobro- } \\
\text { mine }\end{array}$ & 180 & $0.5[23]$ & 820,000 & $3 * 10^{-7}$ & 5.5 & 0.16 \\
$*$ & $\begin{array}{l}\text { Based on the fit presented in Figure 2.B to }(1-2) \\
* *\end{array}$ \\
Using an approximate value for $a$ of $0.5 \mathrm{~cm}$. & & \\
$* * *$ & $\begin{array}{l}\text { Based on the fits presented in Figure 4 } \\
\text { Using (3) }\end{array}$
\end{tabular}

the uncoated films and the ones coated by a colloidal shell is identical, so that comparing the two would allow determination of the effect of the colloidal shell on transport. As shown in Figure 2, the release rate of theophylline and caffeine from the bare alginate hydrogel is similar. In contrast, the release of theobromine requires significantly longer periods, in qualitative agreement with previous studies of the three drug's release from alginate gels [24]. Aspirin has a much faster release rate than all other drugs, in agreement with our previous observations $[17,18]$.

To quantify the transport rate of the three diffusants, the release data were fit to the diffusion model described by (1-2) [18], as shown in Figure (2.B). Since the gels are uncoated, the parameter $L$, which describes the shell/surface resistance, is equal to infinity for all cases [18]. To demonstrate the validity of the model, we collapse the data onto one curve using a normalization factor for the time $t$ that arises from (1-2), namely, $a^{2} / D$, where $a$ is the thickness of the alginate film and $D$ the diffusion coefficient of the drug in the hydrogel core. The model prediction is plotted as a solid line, and has no free parameters when plotted in these coordinates.

The fact that all four release profiles can be successfully collapsed in this manner onto the model prediction is a strong indicator for the model validity in the uncoated gels. The values found by fitting the data are tabulated in Table 1. Unfortunately, we do not have accurate measurements of the film thickness for the gel films (although, since we use the same volume in the same vial for all drugs, both in the case of coated and uncoated gels, it is the same in all experiments).

However, estimating $a$ to be $0.5 \mathrm{~cm}$, a value consistent with our experimental set up, yields the following estimates for the diffusion coefficients of the drugs in our formulation of alginate: $2.5^{*} 10^{-5} \mathrm{~cm}^{2} / \mathrm{s}$ for aspirin, $3.3 * 10^{-6} \mathrm{~cm}^{2} / \mathrm{s}$ for caffeine, $3 * 10^{-6} \mathrm{~cm}^{2} / \mathrm{s}$ for theophylline, and $3 * 10^{-7} \mathrm{~cm}^{2} / \mathrm{s}$ for theobromine. The results for caffeine and theophylline are somewhat lower than the values for the diffusion coefficient of these drugs in water $\left(6.3 * 10^{-6} \mathrm{~cm}^{2} / \mathrm{s}\right.$ [25] for caffeine and $6.15 * 10^{-6} \mathrm{~cm}^{2} / \mathrm{s}$ [26] for theophylline), as may be expected since transport through the hydrogel should be slower than through pure water.

In Figure (3.A) we plot the release of the drugs as measured for alginate hydrogels coated by a self-assembled colloidal monolayer. As may be expected, the presence of the colloidal shell reduces the release rate when compared to the release from uncoated gels. For example, in the uncoated case both caffeine and theophylline release more that $90 \%$ (namely, $f>0.9$ ) after 20,000 sec, while in the coated case the fraction released at that time interval is of order $60 \%$.Also as expected, the 


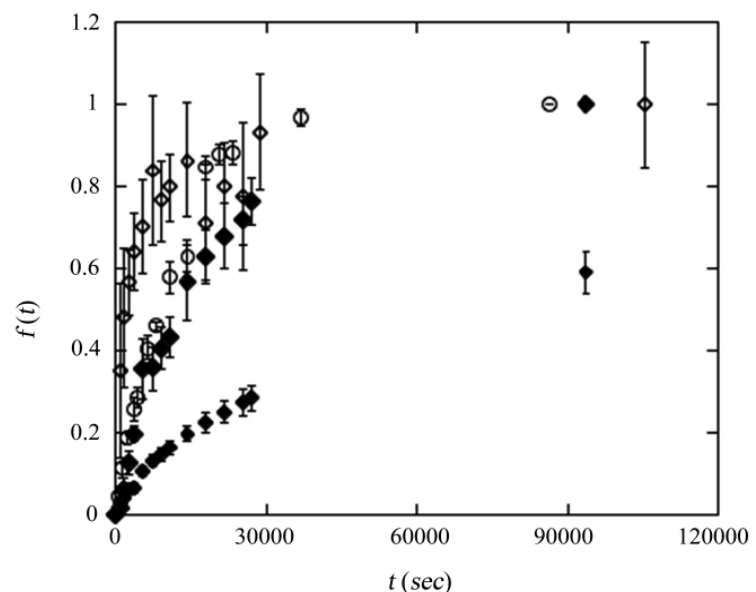

(a)

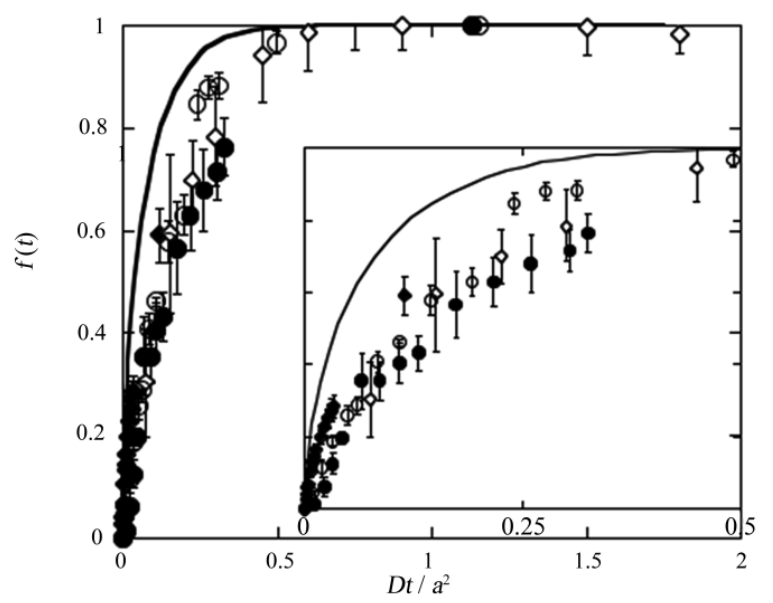

(b)

Figure 3. The release of caffeine, theophylline and theobromine from alginate hydrogels coated by a monolayer of $3.3 \mathrm{~mm}$ colloidal particles. $f$ defines the fraction of the initially encapsulated diffusant, released by time $t$. The symbols are as defined in Figure 2. (A) The fractional release as a function of time; (B) The fractional release as a function of the normalized time $D t / a^{2}$, where the value of $D / a^{2}$ is as calculated from the fit to the uncoated hydrogel (Figure 2.B). The solid line describes the model prediction for the uncoated gels.

relative rates remain the same as in the uncoated case: Aspirin is released most rapidly, theophylline and caffeine have similar rates, and theobromine requires the longest time. However, the raw data cannot distinguish between the effects of the diffusion coefficient in the hydrogel, and the potential effect of the self assembled colloidal monolayer.

To determine the effect of the colloidal shell, we again normalize time the value of $a^{2} / D$ as calculated from the uncoated gels (Figure 3.B). If the effect of the shell is similar for all drugs, then they should again collapse onto the same curve, although this one would differ from that of the uncoated system, as given in (1-3). We see, however, that the normalized release profiles do not overlap: Although all are slower than the predicted rate for the uncoated gels (as depicted by the solid line), caffeine and theobromine seem to have a faster rate than that of aspirin, while theophylline is clearly slower than all other drugs.

The two film model suggests that these differences in the release rates may be due to the packing density of the colloidal particles in the shell; since the shell is self assembled, the density may vary between the different systems. Therefore, we use (1-2) to fit the release profiles of the four drugs to evaluate the value of $L$, the shell transport retardation parameter, and (3) to correlate this parameter to the colloidal packing density. The fits are presented in Figure 4, and the values listed in Table 1.

We find that the values of the shell permeability parameter $L$ fall, for all drugs, in the range of 2-5, which translated to a range for the packing volume fraction of particles in the shell of $0.3-0.16$. For both caffeine and aspirin, the best fit is obtained with a value of $L=3.5$, which translates to a colloidal packing density in the shell using (3) of $\square \approx 0.2$. For theophylline, the best fit is for $L=2$, namely, $\square \approx 0.3$, while for theobromine the best fit is for $L=5.5$, corresponding to $\square \approx 0.16$. While there are clear differences between these values, they are all within the same range for the packing density. Moreover, there is no clear trend linking the drug properties and the colloidal packing density in the shell: Caffeine and aspirin have similar packing densities, despite the differences in structure, MW and solubility. Theophylline, which has a similar solubility to that of aspirin- which is intermediate between caffeine and theobromine- has the highest packing density, while theobromine has the lowest.

\section{Conclusions}

Previous studies have shown that the properties of an encapsulated drug can significantly affect the properties of the encapsulating hydrogel. In this paper we investigate the effect of drugs, encapsulated in hydrogel films, on the self-assembly of a colloidal shell. Fourmodel drugs are investigated: aspirin, caffeine, theophylline and theobromine. These drugs are similar in MW, and three of them (caffeine, theophylline and theobromine) have a similar structure and even similar physiological effects. However, they differ significantly in their solubility in water.

We find that the release profiles in all systems (both uncoated hydrogels and ones coated by the self assembled colloidal shells) can be fit well with a two-film diffusion model [18]. The packing density in the self-assembled colloidal layers is found to vary, between the drugs, in the range of $0.16-0.3$. The lowest packing 

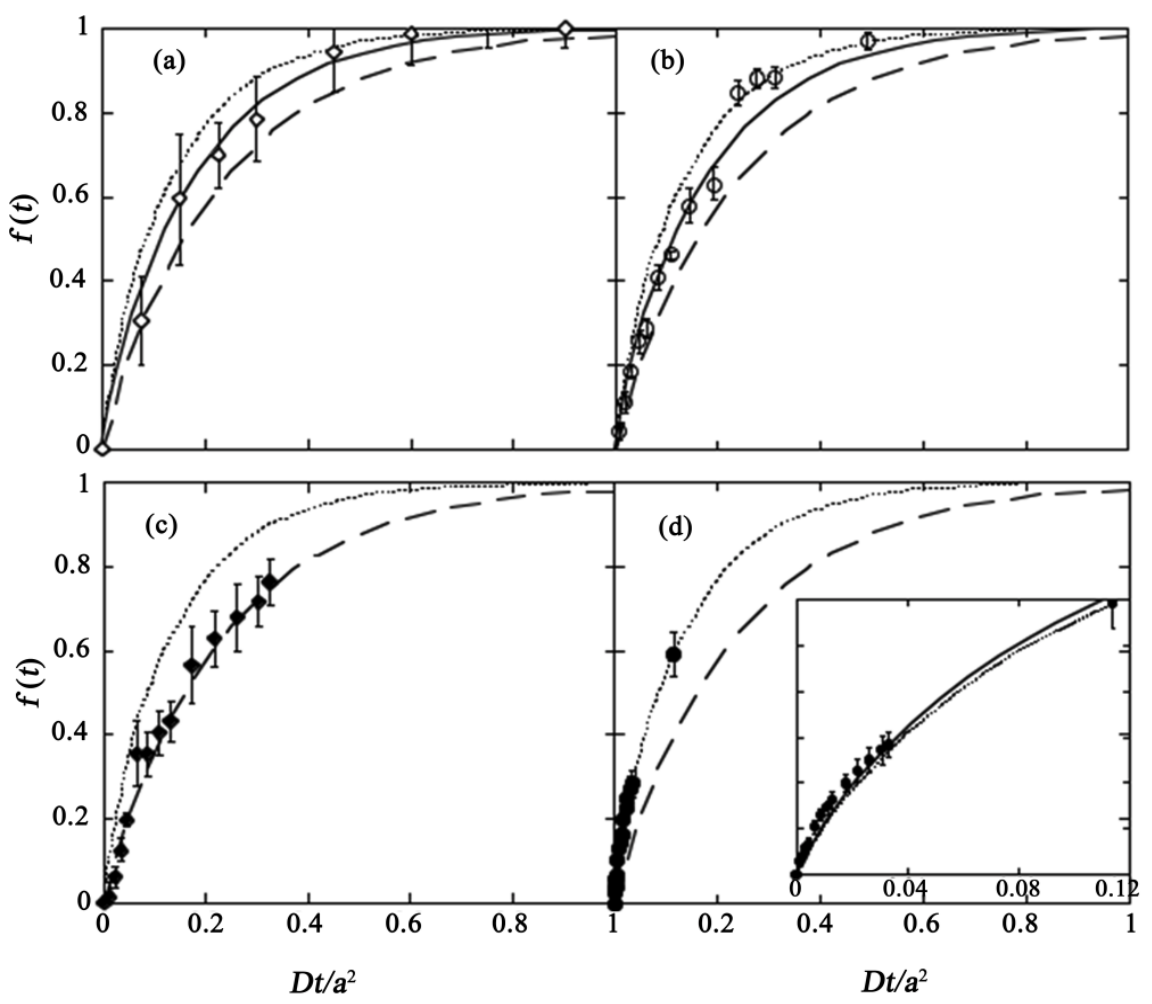

Figure 4. Fitting the release rate from coated hydrogel to the two-film model (equations 1-2). The dotted line is for a shell where $L=5$, and the dashed line where $L=2$. a ) Aspirin. The solid line is the model with a value of $L=3.5$; $b$ ) $C$ affeine. The solid line is the model with a value of $L=3.5$; c) Theophylline; d) Theobromine. The solid line in the inset is for $L=5.5$.

density is found for theobromine, which has the lowest solubility in water. However, the highest packing density is found for theophylline, which hasanintermediate water solubility (see Table 1). Caffeine and aspirin have identical packing densities, despite the fact that the solubility of caffeine in water is three times as high as that of aspirin.

We conclude that there is some effect of the drug properties on the packing density in the self assembled colloidal shells. However, this effect is weak, and cannot be directly linked to the drug properties, in particular solubility in water.

\section{Acknowledgements}

Thanks to NSF/IDBR award number 0649897 for financial support. RTR acknowledges the NSF-IGERT DGE-0654313 fellowship.

\section{REFERENCES}

[1] T. Kaasgaard and T. L. Andresen, "Liposomal Cancer Therapy: Exploiting Tumor Characteristics", Expert Opinion on Drug Delivery, Vol. 7, No. 2 , 2010, pp. 225-243. doi:10.1517/17425240903427940

[2] W. N. Hait, "Targeted Cancer Therapeutics”, Cancer
Research, Vol. 69, No. 4, 2009, pp. 1263-1267 . doi:10.1158/0008-5472.CAN-08-3836

[3] L. Sagalowicz and M. E. Leser, "Delivery Systems for Liquid Food Products", Current Opinion in Colloid and Interface Science, Vol. 15, No. 1-2, 2010, pp. 61-72. doi:10.1016/j.cocis.2009.12.003

[4] X. Wu and R. H. Guy, "Applications of Nanoparticles in Topical Drug Delivery and in Cosmetics”, Journal of Drug Delivery Science and Technology, Vol. 19, No. 6, 2009, pp. 371-384.

[5] J. Pardeike, A. Hommoss and R. H. Muller, "Lipid Nanoparticles (SLN, NLC) in Cosmetic and Pharmaceutical Dermal Products", International Journal of Pharmaceutics, Vol. 366, No. 1-2, 2009, pp 170-184. doi:10.1016/j.ijpharm.2008.10.003

[6] D. S. Pisal, M. P. Kosloski and S. V. Balu-Iyer, "Delivery of Therapeutic Proteins" Journal of Pharmaceutical Sciences, Vol. 99, No. 6, 2010, pp. 2557-2575. doi:10.1002/jps.22054

[7] A. D. Dinsmore, M. F. Hsu, M. G. Nikolaides, M. Marquez, A.R. Bausch and D. A. Weitz, "Colloidosomes: Selectively Permeable Capsules Composed of Colloidal Particles” Science, Vol. 298, No. 5595, 2002, pp. 1006-1009. doi:10.1126/science. 1074868

[8] D. Lee and D. A Weitz, "Double Emulsion-Templatednanoparticlecolloidosomes with Selective Permeability”, Advanced Materials, Vol. 20, No. 18, 
2002, pp. 3498-3503. doi:10.1002/adma.200800918

[9] D. Lee and D.A Weitz, "Nonspherical Colloidosomes with Multiple Compartments from Double Emulsions”, Small, Vol. 5, No. 17, 2009, pp. 1932-1935. doi:10.1002/smll.200900357

[10] S. Shilpi, A. Jain, Y. Gupta and S. K. Jain "Colloidosomes: an Emerging Vesicular System in Drug Delivery”, Critical Reviews in Therapeutic Drug Carrier Systems, Vol. 24, No. 4, 2007, pp. 361-391.

[11] J. W. Kim, A. Fernandez-Nieves A, N. Dan N, A. S. Utada AS, M. Marquez and D. A. Weitz, "Colloidal Assembly Route for Responsive Colloidosomes with Tunable Permeability" Nano Letters, Vol. 7, No. 9, 2007, pp. 2876-2880. doi:10.1021/nl0715948

[12] A. S. Miguel, J. Scrimgeour, J. E. Curtis and S. H. Behrens, "Smart Colloidosomes with a Dissolution Trigger", Soft Matter, Vol. 6, No. 14, 2010, pp. 3163-3166. doi:10.1039/c002930k

[13] R. McGorty, J. Fung, D. Kaz and V. N. Manoharan, "Colloidal Self-Assembly at an Interface”, Materials Today, Vol. 13, No. 6, 2010, pp. 34-42. doi:10.1016/S1369-7021(10)70107-3

[14] M. H. Lee, V. Prasad and D. Lee, "Microfluidic Fabrication of Stable Nanoparticle-Shelled Bubbles”, Langmuir, Vol. 26, No. 4, 2010, pp. 2227-2230. doi:10.1021/la904425v

[15] J. Texter, "Templating Hydrogels", Colloid and Polymer Science, Vol. 287, No. 3, 2009, pp. 313-321. doi:10.1007/s00396-008-1990-z

[16] X. C. Yang and Z. H. Mo "Microcapsules from the Self-Assembly of Nanoparticles at Interfaces”, Progress in Chemistry, Vol. 22, No. 9, 2010, pp. 1735-1740.

[17] R. T. Rosenberg and N. R. Dan, "Controlling Surface Porosity and Release from Hydrogels Using a Colloidal Particle Coating”, Journal of Colloid and Interface Science, Vol. 349, No. 2, 2010, pp. 498-504. doi:10.1016/j.jcis.2010.05.095

[18] R. T. Rosenberg and N. R. Dan, "Diffusion through Colloidosome Shells", Journal of Colloid and Interface Science, 2010. In press.

[19] S. J. Siegel, J. B. Kahn, K. Metzger K, K. I. Winey KI, K. Werner K and N. Dan, "Effect of Drug Type on the De- gradation Rate of PLGA Matrices”, European Journal of Pharmaceutics and Biopharmaceutics, Vol. 64, No. 3, 2006, pp. 287-293. doi:10.1016/j.ejpb.2006.06.009

[20] R. Rosenberg, W. Devenney, S. Siegel and N. Dan, "Anomalous Release of Hydrophilic Drugs from Poly(Epsilon-Caprolactone) Matrices”, Molecular Pharmaceutics, Vol. 4, No. 6. 2007, pp. 943-948. doi:10.1021/mp700097x

[21] A. Meyer, T. Ngiruwonsanga and G. Henze, "Determination of Adenine, Caffeine, Theophylline and Theobromine by HPLC with Amperometric Detection", Journal of Analytical Chemistry, Vol. 356, 1996, pp. 284-287.

[22] I. P. o. C. S. (IPCS), http://www.inchem.org/documents/pims/pharm/aspirin.ht m\#SectionTitle:3.2\%20Chemical\%20structure

[23] J. B. Thomas, J. H. Yen, M. M. Schantz, B. J. Porter, and K.E. Sharpless, "Determination of Caffeine, Theobromine, and Theophylline in Standard Reference Material 2384, Baking Chocolate, Using Reversed-Phase Liquid Chromatography”, Journal of Agricultural and Food Chemistry, Vol. 52, No. 11, 2004, pp. 3259-3263. doi:10.1021/jf030817m

[24] P. Sriamornsak and R. A. Kennedy, "Effect of Drug Solubility on Release Behavior of Calcium Polysaccharide Gel-coated Pellets”, European Journal of Pharmaceutical Sciences, Vol. 32, No. 3, 2007, pp. 231-239. doi:10.1016/j.ejps.2007.08.001

[25] D. W. Green and R. H. Perry, "Perry's Chemical Engineers' Handbook”, 8th ed., McGraw-Hill, Columbus, 2008.

[26] M. Grassi, I. Colombo and R. Lapasin, "Experimental Determination of the Theophylline Diffusion Coefficient in Swollen Sodium-Alginate Membranes", Journal of Controlled Release, Vol. 76, No. 1-2, 2001, pp. 93-105. doi:10.1016/S0168-3659(01)00424-2

[27] R. A. Thakur and B. B. Michniak, “Transdermal and Buccal Delivery of Methylxanthines Through Human Tissue In Vitro", Drug Development and Industrial Pharmacy, Vol. 33, No. 5, 2007, pp. 513-521. doi:10.1080/03639040600901994 